\title{
QUASICONFORMAL HOMOGENEITY AFTER GEHRING AND PALKA
}

\author{
PETRA BONFERT-TAYLOR, RICHARD CANARY AND EDWARD TAYLOR
}

\section{IntroduCtion}

In a very influential paper [16] Gehring and Palka introduced the notions of quasiconformally homogeneous and uniformly quasiconformally homogeneous subsets of $\overline{\mathbb{R}^{n}}$. Their motivation was to provide a characterization of quasi-disks, i.e. domains in $\overline{\mathbb{R}^{n}}$ which are quasiconformally homeomorphic to the unit disk in $\mathbb{R}^{n}$. (This paper also introduced the important concept of the quasihyperbolic metric on a domain in $\overline{\mathbb{R}^{n}}$.) As a generalization, Bonfert-Taylor, Canary, Martin and Taylor [5] initiated the study of uniformly quasiconformally homogeneous hyperbolic manifolds.

In this paper, we review the theory of quasiconformally homogeneous subsets of $\overline{\mathbb{R}^{n}}$ and uniformly quasiconformally homogeneous hyperbolic manifolds. We finish with a discussion of open problems in the theory.

The authors have all benefitted from the inspiration, mathematical and personal, of Fred Gehring's mathematical career. Petra BonfertTaylor was a postdoctoral assistant professor under Fred's supervision, Dick Canary was a colleague for many years and Ed Taylor was also a postdoctoral assistant professor at the University of Michigan. It is our pleasure to review the impact of one of Fred Gehring's papers on the field.

\section{QuAsiconformally HomogeneOUs Subsets of $\overline{\mathbb{R}^{n}}$}

A domain $D \subset \overline{\mathbb{R}^{n}}$ is said to be quasiconformally homogeneous if for all $x, y \in D$, there exists a quasiconformal map $f: D \rightarrow D$ such that $f(x)=y$. In their paper, Gehring and Palka [16, Lemma 3.2] observed that every domain is quasiconformally homogenous. We will sketch the proof as variations of the proof will appear later.

Proposition 2.1. (Gehring-Palka [16, Lemma 3.2]) Every domain in $\overline{\mathbb{R}^{n}}$ is quasiconformally homogeneous.

Richard Canary was partially supported by NSF grant DMS -1306992 . 
Sketch of proof: The proof is based on the following simple lemma which is proved by normalizing and exhibiting an explicit quasiconformal map.

Lemma 2.2. (Gehring-Palka [16, Lemma 3.1]) If $B$ is a round ball in $\overline{\mathbb{R}^{n}}$ and $a, b \in B$, then there exists a $K$-quasiconformal map $f: \overline{\mathbb{R}^{n}} \rightarrow \overline{\mathbb{R}^{n}}$ such that $f(a)=b,\left.f\right|_{\overline{\mathbb{R}^{n}}-B}=i d$, and

$$
\log K=d_{B}(a, b)
$$

where $d_{B}$ is the Poincaré metric on $B$.

If now $D$ is an arbitrary domain in $\mathbb{R}^{n}$ and $x$ and $y$ lie in $D$, then there exists a finite collection $\left\{B_{i}\right\}_{i=0}^{n}$ of open round balls in $D$ such that $x \in B_{0}, y \in B_{n}$ and $B_{i-1} \cap B_{i}$ is non-empty for all $i$. We then choose $x_{i} \in B_{i-1} \cap B_{i}$ for all $i=1, \ldots, n-1$ and set $x_{0}=x$ and $x_{n}=y$. Lemma 2.2 implies that for all $i=1, \ldots, n$ there exists a quasiconformal map $f_{i}: D \rightarrow D$ such that $f\left(x_{i-1}\right)=x_{i}$. So, if $f=f_{n} \circ \cdots \circ f_{1}$, then $f: B \rightarrow B$ is quasiconformal and $f(x)=y$.

Thus, it is natural to require that there is a uniform upper bound on the dilatation of the quasiconformal map. A domain $D \subset \overline{\mathbb{R}^{n}}$ is said to be $K$-quasiconformally homogeneous if for all $x, y \in D$, there exists a $K$-quasiconformal map $f: D \rightarrow D$ such that $f(x)=y$. If $D$ is $K$-quasiconformally homogeneous for some $K$, we say that it is uniformly quasiconformally homogeneous.

A domain $D \subset \overline{\mathbb{R}^{n}}$ is a $K$-quasi-disk if there exists a $K$-quasiconformal homeomorphism $f: \overline{\mathbb{R}^{n}} \rightarrow \overline{\mathbb{R}^{n}}$ such that $f\left(\Delta^{n}\right)=D$ where $\Delta^{n}$ is the unit disk in $\mathbb{R}^{n}$. Gehring and Palka observed that every $K$-quasi-disk is $K^{2}$-quasiconformally homogeneous. They further show

Theorem 2.3. (Gehring-Palka [16, Theorem 5.5]) If $D \subset \overline{\mathbb{R}^{n}}$ is a uniformly quasiconformally homogeneous domain with boundary $\partial D$, then either

(1) $\partial D$ consists of 0,1 or 2 points,

(2) $\partial D$ is a non-degenerate continuum, or

(3) every neighborhood of every point in $\partial D$ contains infinitely many components of $\partial D$.

Since all simply connected domains in the plane are conformally equivalent to the unit disk, one must further strengthen these conditions to obtain a characterization of quasi-disks. One says that a subset $R \subset \overline{\mathbb{R}^{n}}$ is $K$-ambiently quasiconformally homogeneous if there exists $K>0$ such that for all $x, y \in R$, there exists a $K$-quasiconformal map $f: \overline{\mathbb{R}^{n}} \rightarrow \overline{\mathbb{R}^{n}}$ such that $f(R)=R$ and $f(x)=y$. It is said to 
be ambiently quasiconformally homogeneous if it is $K$-ambiently quasiconformally homogeneous for some $K$. Notice that here our subsets need not be domains.

Gehring and Palka constructed the first non-trivial examples of ambiently quasiconformally homogeneous domains by observing that any component of the domain of discontinuity of a convex cocompact subgroup of $\operatorname{Isom}_{+}\left(\mathbb{H}^{n+1}\right)$ is an ambiently quasiconformally homogeneous domain in $\overline{\mathbb{R}^{n}}=\partial \mathbb{H}^{n+1}$ (see [16, Lemma 4.3]). In particular, by considering Schottky groups, they showed that there exists an uniformly quasiconformally homogeneous domain in $\overline{\mathbb{R}^{n}}$ whose complement is a Cantor set (see [16, Example 4.4]). (In each case, Gehring and Palka only claim uniform quasiconformal homogeneity, but the proofs they offer immediately establish ambient quasiconformal homogeneity.)

Erkama [12] proved that a Jordan curve in the plane is ambiently quasiconformally homogeneous if and only if it is a quasi-circle (i.e. it is the image of the unit circle under a quasiconformal map of $\widehat{\mathbb{C}}$ ). Brechner and Erkama [10] extended this result from Jordan curves to non-degenerate continua.

Sarvas [27] obtained a characterization of quasi-disks.

Theorem 2.4. (Sarvas [27]) A domain $D \subset \overline{\mathbb{R}}^{2}$ is a quasidisk if and only if it is an ambiently quasiconformally homogeneous Jordan domain.

Sketch of Proof: Gehring and Palka [16] observed that $K$-quasi-disks are $K^{2}$-ambiently quasiconformally homogeneous, since the unit disk is ambiently conformally homogeneous and the product of two $K$ quasiconformal maps is $K^{2}$-quasiconformal.

Now suppose that a Jordan domain $D$ is not a quasi-disk, but is $K$-ambiently quasiconformally homogeneous. We may assume that $D$ is a bounded domain in $\mathbb{C}$. Ahlfors [2] showed that if $D$ is not a quasidisk, then there exists a sequence $\left\{\left(u_{n}, v_{n}, w_{n}\right)\right\}$ of triples of distinct points in $\partial D$ so that if $J_{n}$ and $J_{n}^{\prime}$ are the components of $\partial D-\left\{u_{n}, v_{n}\right\}$, and $\operatorname{diam}\left(J_{n}\right) \leq \operatorname{diam}\left(J_{n}^{\prime}\right)$, then $w_{n} \in J_{n}$ and

$$
\lim \frac{\left|w_{n}-v_{n}\right|}{\left|u_{n}-v_{n}\right|}=\infty
$$

We may pass to a subsequence, and possibly choose new triples $\left\{\left(u_{n}, v_{n}, w_{n}\right)\right\}$, so that either (a) the open line segment $\left(u_{n}, v_{n}\right)$ and the round half-disk $D_{n}$ with partial boundary $\left(u_{n}, v_{n}\right)$ (on the same side of $\left(u_{n}, v_{n}\right)$ as $\left.J_{n}\right)$ are both contained in $D$ for all $n$, or (b) $\left(u_{n}, v_{n}\right)$ is contained in $\mathbb{C}-D$ for all $n$. 
In case (a), fix a point $a \in D$ and let $f_{n}: \widehat{\mathbb{C}} \rightarrow \widehat{\mathbb{C}}$ be a $K$-quasiconformal map so that $f_{n}(D)=D$ and $f_{n}(a)=y_{n}$ where $y_{n}$ is the point in $D_{n}$ midway "above" the midpoint of $\left(u, v_{n}\right)$. Let $L_{n}: \widehat{\mathbb{C}} \rightarrow \widehat{\mathbb{C}}$ be the extension of an affine map of $\mathbb{C}$ so that $L_{n}\left(u_{n}\right)=-1, L_{n}\left(v_{n}\right)=1$ and $L_{n}\left(y_{n}\right)=\frac{1}{2} i$. Then, $\left\{g_{n}=L_{n} \circ f_{n}\right\}$ is a normal family, since $g_{n}(D)$ always misses -1 , 1 and $\infty$, so, up to subsequence, it converges to a $K$-quasiconformal map $g: \widehat{\mathbb{C}} \rightarrow \widehat{\mathbb{C}}$. (Notice that $g$ must be non-constant, since $g_{n}(a)=\frac{1}{2} i$ for all $n$ and $\left\{\operatorname{diam}\left(g_{n}(D)\right)\right\} \rightarrow \infty$.) Now, for each $n$, choose $z_{n} \in J_{n}^{\prime}$ so that $\left|z_{n}-v_{n}\right| \geq \frac{1}{2}\left|w_{n}-v_{n}\right|$, and pass to a subsequence so that the following limits all exist: $\lim f_{n}^{-1}\left(u_{n}\right)=\hat{u}, \lim f_{n}^{-1}\left(v_{n}\right)=\hat{v}, \lim f_{n}^{-1}\left(w_{n}\right)=\hat{w}$ and $\lim f_{n}^{-1}\left(z_{n}\right)=\hat{z}$. Then $g(\hat{u})=-1, g(\hat{v})=1$ and $g(\hat{w})=g(\hat{z})=\infty$ (since $\lim \frac{\left|w_{n}-v_{n}\right|}{\left|u_{n}-v_{n}\right|}=\infty$ ). This is impossible since it implies that $\hat{u}, \hat{v}$, and $\hat{w}$ are all distinct, but $\hat{z}=\hat{w}$. However, $\hat{w}$ and $\hat{z}$ lie in distinct components of $\partial D-\{\hat{u}, \hat{v}\}$. The argument to handle case (b) uses similar techniques.

Hjelle [20] showed that the assumption that $D$ is a Jordan domain is necessary in Sarvas' theorem by giving an example of an ambiently quasiconformally homogeneous simply connected subset of $\widehat{\mathbb{C}}$ which is not a quasi-disk. The domain of discontinuity of a purely hyperbolic degenerate group also provides such an example (see Bonfert-TaylorCanary-Martin-Taylor-Wolf [6, Theorem 1.5]).

MacManus, Näkki, and Palka [23, Theorem 3.1] characterize the possible topological types of ambiently quasiconformally homogeneous compact subsets of $\widehat{\mathbb{C}}$.

Theorem 2.5. (MacManus-Näkki-Palka [23, Theorem 3.3]) If $R$ is an ambiently quasiconformally homogeneous compact subset of $\widehat{\mathbb{C}}$, then either

(1) $R=\widehat{\mathbb{C}}$

(2) $R$ is a finite set of points,

(3) $R$ is a finite union of disjoint quasicircles bounding a domain in $\widehat{\mathbb{C}}$, or

(4) $R$ is a Cantor set of Hausdorff dimension less than 2.

All sets of type (a), (b) and (c) are ambiently quasiconformally homogeneous, but there is no known characterization of which Cantor sets are ambiently quasiconformally homogeneous. However, they show that the middle- $\frac{1}{3}$-Cantor set (see [23, Example 3.5]) and limit sets of Schottky groups (see [24, Theorem 1.2]) are ambiently quasiconformally 
homogeneous. Therefore, there exist ambiently quasiconformally homogeneous Cantor sets with any Hausdorff dimension in $(0,2)$ (see also Gong-Martin [18]).

MacManus, Näkki, and Palka [24] further define a subset $E \subset \widehat{\mathbb{C}}$ to be uniformly quasiconformally bi-homogeneous if there exists $K$ so that if $(a, b),(c, d) \in E \times E^{c}$ then there exists a $K$-quasiconformal map $f: \widehat{\mathbb{C}} \rightarrow \widehat{\mathbb{C}}$ such that $f(E)=E, f(a)=c$ and $f(b)=d$. They show $[24$, Theorem B] that a non-empty compact subset of $\widehat{\mathbb{C}}$ is uniformly quasiconformally bi-homogeneous if and only if it either (a) consists of at most two points, (b) is a quasi-circle or (c) is an image of the middle- $\frac{1}{3}$ Cantor set under a quasiconformal homeomorphism of $\widehat{\mathbb{C}}$. They further show [24, Theorem D] that a Cantor set $E$ is uniformly quasiconformally bi-homogeneous if and only if $E$ is uniformly perfect and $E^{c}$ is a uniform domain.

Bonfert-Taylor and Taylor [9, Theorem 1.1] show that if $E$ is a Cantor set in $\widehat{\mathbb{C}}$ and both $E$ and its complement $E^{c}$ are ambiently quasiconformally homogeneous, then $E$ is quasiconformally bi-homogenous. Therefore, $E$ must be uniformly perfect and $E^{c}$ must be a uniform domain. Moreover, they exhibit Cantor sets $E$ and $F$ such that (a) $E$ is ambiently quasiconformally homogeneous and $E^{c}$ is not (see [9, Example 3.3]), and (b) $F$ is not ambiently quasiconformally homogeneous, but $F^{c}$ is (see [9, Example 3.1]).

\section{UNIFORMLY QUASICONFORMALLY HOMOGENEOUS HYPERBOLIC MANIFOLDS}

Inspired by the work of Gehring and Palka [16], Bonfert-Taylor, Canary, Martin and Taylor [5] initiated the study of uniformly quasiconformally homogeneous hyperbolic manifolds. In this paper, all manifolds will be orientable.

A (complete) hyperbolic manifold $N=\mathbb{H}^{n} / \Gamma$ is said to be $K$-quasiconformally homogeneous if for all $x, y \in N$, there exists a $K$-quasiconformal homeomorphism $f: N \rightarrow N$ such that $f(x)=y$. It is said to be uniformly quasiconformally homogeneous if it is $K$-quasiconformally homogeneous for some $K$. We define the quasiconformal homogeneity constant of $N$ to be

$$
K(N)=\min \{K \mid N \text { is } K \text {-quasiconformally homogeneous }\} .
$$

It is an immediate consequence of compactness theorems for families of $K$-quasiconformal maps that we may take minimum, rather than simply infimum, in this definition (see [5, Lemma 2.1]). 
One may use the geometry of quasiconformal homeomorphisms to obtain some basic restrictions on the geometry of uniformly quasiconformally homogeneous hyperbolic manifolds. Let $\ell(N)$ denote the infimum of the set of lengths of homotopically non-trivial closed curves in $N$ and let $d(N)$ denote the supremum of the set of diameters of embedded hyperbolic balls in $N$.

Theorem 3.1. (Bonfert-Taylor-Canary-Martin-Taylor [5, Theorem 1.1]) For all $n \geq 2$ and $K \geq 1$, there exists $m(n, K)>0$ such that if $N=\mathbb{H}^{n} / \Gamma$ is a $K$-quasiconformally homogeneous hyperbolic n-manifold, other than $\mathbb{H}^{n}$, then

(1) $\ell(N)$ is positive and $d(N)$ is finite. In particular,

$$
d(N) \leq K \ell(N)+2 K \log 4 .
$$

(2) $\ell(N) \geq m(n, K)$, and

(3) every non-trivial element of $\Gamma$ is hyperbolic and the limit set $\Lambda(\Gamma)$ of $\Gamma$ is all of $\partial \mathbb{H}^{n}$.

Sketch of proof: Suppose that $x$ lies on a closed homotopically nontrivial curve $\alpha$ of length $\ell$ and that $y$ is the center of an embedded hyperbolic ball $B$ of radius $r$. Let $f: N \rightarrow N$ be a $K$-quasiconformal homeomorphism such that $f(x)=y$. Since every $K$-quasiconformal homeomorphism is a $(K, K \log 4)$-quasi-isometry (see Vuorinen [29, Theorem 11.2]) and there exists $z \in \alpha$ such that $f(z)$ is not contained in $B$ (since $f(\alpha)$ is homotopically non-trivial), we see that

$$
r \leq d(f(x), f(z)) \leq K d(x, z)+K \log 4 \leq K l / 2+K \log 4
$$

and (1) follows.

Since there is a uniform positive lower bound $d_{n}$ on $d(N)$ which depends only on $n$, property (2) follows similarly from the fact, again see [29, Theorem 11.2], that if $f$ is $K$-quasiconformal, then

$$
\tanh \left(\frac{d(f(x), f(y))}{2}\right) \leq \lambda_{n}^{1-J}\left(\tanh \left(\frac{d(x, y)}{2}\right)\right)^{J}
$$

where $J=K^{1 /(1-n)}$ and $\lambda_{n} \in\left[4,2 e^{n-1}\right]$ is the Grötzsch constant. In particular, one may take $m(n, K)=2 \tanh ^{-1}\left(\lambda_{n}^{J-1} \tanh \left(d_{n} / 2\right)^{1 / J}\right)$.

Since $\ell(N)>0, \Gamma$ cannot contain parabolic elements and since $d(N)$ is finite, the limit set $\Lambda(\Gamma)$ must be all of $\partial \mathbb{H}^{n}$. Property (3) follows.

Gehring and Palka's proof of Proposition 2.1 may be easily adapted to show that every closed hyperbolic manifold is uniformly quasiconformally homogeneous. If one keeps careful track of the constants one obtains: 
Proposition 3.2. (Bonfert-Taylor-Canary-Martin-Taylor [5, Proposition 2.4]) Every closed hyperbolic $n$-manifold $N$ is uniformly quasiconformally homogeneous. Moreover,

$$
K(N) \leq\left(e^{\frac{\ell(N)}{4}}+1\right)^{2(n-1)\left(\frac{4 \operatorname{diam}(N)}{\ell(N)}+1\right)}
$$

where $\operatorname{diam}(N)$ is the diameter of $N$.

A similar argument shows that:

Proposition 3.3. (Bonfert-Taylor-Canary-Martin-Taylor [5, Proposition 2.7]) Every regular cover of a closed hyperbolic orbifold $N$ is uniformly quasiconformally homogeneous.

As a consequence of Theorem 3.1 and Proposition 3.2 one see that a geometrically finite hyperbolic manifold is uniformly quasiconformally homogeneous if and only if it is closed (see [5, Corollary 1.2]).

We now discuss rigidity phenomena for uniformly quasiconformally homogeneous hyperbolic $n$-manifolds where $n \geq 3$. The key tool is McMullen's version of Sullivan's rigidity theorem.

Theorem 3.4. (McMullen [26, Theorem 2.10]) Suppose that $N=\mathbb{H}^{n} / \Gamma$ is a hyperbolic $n$-manifold where $n \geq 3$ and there is an upper bound on the radius of an embedded hyperbolic ball in $N$. If $f: N \rightarrow N$ is a quasiconformal homeomorphism, then $f$ is homotopic to an orientationpreserving isometry.

We may then combine Proposition 3.3, Theorem 3.1 and Theorem 3.4 to show that:

Theorem 3.5. (Bonfert-Taylor-Canary-Martin-Taylor [5, Theorem 1.3]) If $n \geq 3$, a hyperbolic $n$-manifold is uniformly quasiconformally homogeneous if and only if it is a regular cover of a closed hyperbolic orbifold.

Sketch of proof: We may assume that $N \neq \mathbb{H}^{n}$, since the result is clearly true when $N=\mathbb{H}^{n}$. Proposition 3.3 shows that all regular covers of closed hyperbolic orbifolds are uniformly quasiconformally homogeneous.

Now suppose that $N=\mathbb{H}^{n} / \Gamma$ is $K$-quasiconformally homogeneous. Recall that if $N=\mathbb{H}^{n} / \Gamma$ is a hyperbolic manifold and the limit set $\Lambda(\Gamma)$ of $\Gamma$ contains more than three points, then the group $\Theta$ of orientationpreserving isometries of $N$ acts properly discontinuously on $N$. Therefore, since $\Lambda(\Gamma)=\partial \mathbb{H}^{n}$, by Theorem 3.1, $N$ is a regular cover of the hyperbolic orbifold $N / \Theta$. We will observe that $N / \Theta$ has bounded diameter, so is closed. 
If $x, y \in N$, then there exists a $K$-quasiconformal map $f: N \rightarrow N$ such that $f(x)=y$. McMullen's Rigidity Theorem 3.4 implies that there exists an orientation-preserving isometry $g: N \rightarrow N$ which is homotopic to $f$. We may then choose lifts $\tilde{f}: \mathbb{H}^{n} \rightarrow \mathbb{H}^{n}$ and $\tilde{g}: \mathbb{H}^{n} \rightarrow \mathbb{H}^{n}$, such that $\varphi=\tilde{g}^{-1} \circ \tilde{f}$ is $K$-quasiconformal and $\varphi$ extends to the identity map on $\Lambda(\Gamma)=\partial \mathbb{H}^{n}$.

The family of $K$-quasiconformal homeomorphisms of $\mathbb{H}^{n}$ which restrict to the identity on $\partial \mathbb{H}^{n}$ is compact, so one sees immediately that:

Lemma 3.6. ([5, Lemma 4.1]) For all $n \geq 2$, there exists an increasing function $\psi_{n}:(1, \infty) \rightarrow(0, \infty)$ such that if $\varphi: \mathbb{H}^{n} \rightarrow \mathbb{H}^{n}$ is $K$-quasiconformal and extends to the identity on $\partial \mathbb{H}^{n}$, then

$$
d(x, \varphi(x)) \leq \psi_{n}(K)
$$

for all $x \in \mathbb{H}^{n}$. Moreover, $\lim _{K \rightarrow 1^{+}} \psi_{n}(K)=0$.

Lemma 3.6 then implies that $d(y, g(x)) \leq \psi_{n}(K)$. Therefore, $N / \Theta$ has diameter at most $\psi_{n}(K)$, so $N$ is a regular cover of the closed hyperbolic orbifold $N / \Theta$.

Since, for all $n$, there is a uniform positive lower bound $r_{n}$ on the diameter of a closed hyperbolic $n$-orbifold, and $\lim _{K \rightarrow 1^{+}} \psi_{n}(K)=0$, we see that there is a uniform lower bound on the quasiconformal homogeneity constant of a hyperbolic $n$-manifold other than $\mathbb{H}^{n}$.

Theorem 3.7. (Bonfert-Taylor-Canary-Martin-Taylor [5, Theorem 1.4]) If $n \geq 3$, there exists $K_{n}>1$ such that if $N$ is a uniformly quasiconformally homogeneous hyperbolic n-manifold other than $\mathbb{H}^{n}$, then

$$
K(N) \geq K_{n}
$$

One can completely characterize uniformly quasiconformally hyperbolic homogeneous hyperbolic 3-manifolds with finitely generated fundamental group.

Theorem 3.8. (Bonfert-Taylor-Canary-Martin-Taylor [5, Theorem 7.1]) If $N$ is a non-compact uniformly quasiconformally homogeneous hyperbolic 3-manifold with finitely generated fundamental group, then there exists a closed hyperbolic 3-manifold $M$ which fibers over the circle such that $N$ is the cover of $M$ associated to the fiber.

\section{QuASICONFORMALly HOMOGENEOUS SURFACES}

It is natural to ask whether Theorems 3.5 and 3.7 generalize to the setting of hyperbolic surfaces. Since every diffeomorphism of a closed 
hyperbolic surface is quasiconformal, it is clear that McMullen's rigidity Theorem 3.4 fails for hyperbolic surfaces. Therefore, the proofs outlined in the last section do not extend.

We first review attempts to address the following question:

Question 1: Does there exists $K_{2}>1$ such that if $S$ is a uniformly quasiconformally homogeneous surface, then $K(S) \geq K_{2}$ ?

Kwakkel and Markovic [22] have resolved the question for planar surfaces. (Bonfert-Taylor, Canary, Martin, Taylor and Wolf [6] had earlier produced a lower bound, greater than 1, on the ambient quasiconformal homogeneity constant of a planar hyperbolic surface.)

Theorem 4.1. (Kwakkel-Markovic [22]) There exists $K_{\text {planar }}>1$ so that if $S$ is a planar uniformly quasiconformally hyperbolic surface, then

$$
K(S) \geq K_{\text {planar }}
$$

Bonfert-Taylor, Bridgeman, Canary and Taylor [4] exhibited a lower bound on the uniform quasiconformal homogeneity constant of any hyperelliptic surface. We recall that a closed hyperbolic surface $S$ of genus $g$ is hyperelliptic if it admits a conformal involution with $2 g+2$ fixed points. Hyperelliptic surfaces are known to form a $(2 g-1)-$ complex dimensional subvariety of the Moduli space $M_{g}$ of all (isometry classes of) closed hyperbolic surfaces of genus $g$.

Theorem 4.2. (Bonfert-Taylor-Bridgeman-Canary-Taylor [4]) There exists a constant $K_{\text {hyp }}>1$, such that if $S$ is a closed hyperelliptic hyperbolic surface, then

$$
K(S) \geq K_{\text {hyp }}
$$

Sketch of proof: If not, there exists a sequence $\left\{S_{j}\right\}$ of closed hyperelliptic surfaces such that $\lim K\left(S_{j}\right)=1$. We may assume that $\left\{S_{j}\right\}$ converges geometrically to a surface $S_{\infty}$. It is easy to check, using compactness results for families of quasiconformal maps, that $K\left(S_{\infty}\right)=1$, so that $S_{\infty}=\mathbb{H}^{2}$. In particular, $\lim \ell\left(S_{j}\right)=+\infty$.

We next observe that on any hyperelliptic surface there exist disjoint embedded hyperbolic balls of radius $\frac{l\left(S_{j}\right)}{4}$ about each fixed point of the hyperbolic involution $\varphi_{j}: S_{j} \rightarrow S_{j}$. The balls are embedded by definition of $\ell\left(S_{j}\right)$, so it remains to check that they are disjoint. If $\beta$ is a geodesic arc joining any two fixed points, then $\beta \cup \varphi(\beta)$ is a closed geodesic, so $\beta$ has length at least $\ell\left(S_{j}\right) / 2$. Since $S_{j}$ has $2 g_{j}+2$ fixed point, where $g_{j}$ is the genus of $S_{j}$,

$$
\left(2 g_{j}+2\right) \operatorname{area}\left(B\left(\ell\left(S_{j}\right) / 4\right)\right) \leq \operatorname{area}\left(S_{j}\right)=4 \pi\left(g_{j}-1\right)
$$


where $B\left(\ell\left(S_{j}\right) / 4\right)$ is the ball of radius of $\ell\left(S_{j}\right) / 4$ in $\mathbb{H}^{2}$. Therefore, there is an upper bound on $\ell\left(S_{j}\right)$ which is a contradiction.

More generally, we say that a closed surface $S$ of genus $g$ is $c$-fixed point full, for $c \in(0,2]$, if there exists a non-trivial conformal automorphism of $S$ having $c(g+1)$ fixed points. In particular, every hyperelliptic surface is 2-fixed point full. The argument outlined above easily generalizes to show:

Theorem 4.3. (Bonfert-Taylor-Bridgeman-Canary-Taylor [4]) For each $c \in(0,2]$ there exists $K_{c}>1$ so that if $S$ is a $c$-fixed point full closed hyperbolic surface, then $K \geq K_{c}$.

One may also modify the question by considering more restrictive forms of quasiconformal homogeneity, where the arguments of the previous section do apply. Bonfert-Taylor, Bridgeman, Canary and Taylor [4] define a hyperbolic surface $S$ to be $K$-strongly quasiconformally homogenous if for any $x, y \in S$ there exists a $K$-quasiconformal homeomorphism $f: S \rightarrow S$ such that $f(x)=y$ and $f$ is homotopic to a conformal automorphism of $S$. Similarly $S$ is $K$-extremely quasiconformally homogenous if for any $x, y \in S$ there exists a $K$-quasiconformal homeomorphism $f: S \rightarrow S$ such that $f(x)=y$ and $f$ is homotopic to the identity. Gehring and Palka's Lemma 2.2 can be again used to show that every closed hyperbolic surface is both strongly and extremely quasiconformally homogeneous

We denote the strong quasiconformal homogeneity constant of a surface $S$ by $K_{\text {aut }}(S)$ and the extreme quasiconformal homogeneity constant of the surface by $K_{0}(S)$. The following relationships are immediate (see [4, Lemma 6.1]):

(1) If $S$ is extremely quasiconformally homogeneous, then $S$ is also strongly quasiconformally homogeneous, and

$$
K_{0}(S) \geq K_{\text {aut }}(S) \geq K(S) .
$$

(2) If $S$ is strongly quasiconformally homogeneous, then

$$
K_{\text {aut }}(S) \geq K(S) \text {. }
$$

Lemma 3.6 implies that a hyperbolic surface is extremely quasiconformally homogeneous if and only if it is closed (see [4, Theorem 6.4]). The proofs of Theorems 3.5 and 3.7 immediately generalize in the strongly quasiconformally homogeneous setting. In order to obtain explicit bounds, we note that Yamada [31] proved that

$$
\tau=\sinh ^{-1}\left(\frac{4 \cosh ^{2}(\pi / 7)-3}{8 \cos (\pi / 7)+7}\right) \approx 0.13 .1467
$$


is a lower bound for the diameter of a closed hyperbolic 2-orbifold and that Proposition 6.2 in [4] provides an explicit formula for $\psi_{2}^{-1}$ in terms of the modulus of the Grötsch ring, where $\psi_{2}$ is the function from Lemma 3.6.

Theorem 4.4. (Bonfert-Taylor-Bridgeman-Canary-Taylor [4, Theorem 6.5]) A hyperbolic surface is strongly quasiconformally homogeneous if and only if it is a regular cover of a closed hyperbolic orbifold. Moreover, if $S$ is a strongly quasiconformally homogeneous surface, other than $\mathbb{H}^{2}$, then

$$
K_{\text {aut }}(S) \geq \psi_{2}^{-1}(\tau) \approx 1.0595
$$

Remark: Theorem 6.4 in [4] shows that if $S$ is a closed hyperbolic surface, then

$$
K_{0}(S) \geq \psi_{2}^{-1}\left(\sinh ^{-1}(\sqrt{2} / 3)\right) \approx 1.626
$$

Bonfert-Taylor, Martin, Reid and Taylor [8] obtained a sharp version of Theorem 4.4. They begin by using the isodiametric inequality to show that the $(2,3,7)$-triangle orbifold, denoted $O_{\text {min }}$, has minimal diameter among all hyperbolic two-orbifolds (see [8, Proposition 2.2]).

Theorem 4.5. (Bonfert-Taylor-Martin-Reid-Taylor [8, Theorem 2.3]) If $S$ is a strongly quasiconformally homogeneous hyperbolic surface, other than $\mathbb{H}^{2}$, then

$$
K_{\text {aut }}(S)>\overline{K_{\text {aut }}}=\psi_{2}^{-1}\left(\operatorname{diam}\left(O_{\text {min }}\right)\right) \approx 1.36138 .
$$

Moreover, there exists a sequence $\left\{S_{j}\right\}$ of regular manifold covers of $O_{\text {min }}$ such that $\lim K\left(S_{j}\right)=\overline{K_{a u t}}$.

Sketch of proof: The argument in the previous section establishes that if $S$ is a strongly quasiconformally homogeneous surface, then

$$
K_{\text {aut }}(S) \geq \psi_{2}^{-1}\left(\operatorname{diam}\left(O_{\min }\right)\right) .
$$

The proof that the inequality is strict requires a detailed analysis of the extremal maps for Lemma 3.6. The extremal map is unique and one demonstrates, via the line element field of this map, that it can not be realized as a quasiconformal deformation of any non-elementary Fuchsian group. The reader is referred to [8] for details.

One may show that if $\left\{S_{j}\right\}$ is a sequence of regular manifold covers of $O_{\text {min }}$ such that $\lim \ell\left(S_{j}\right)=+\infty$, then $\lim K\left(S_{j}\right)=\overline{K_{a u t}}$. (The existence of such a sequence of covers is guaranteed by the fact that finitely generated Fuchsian groups are residually finite.) One may assume that 
$\ell\left(S_{j}\right)>>\operatorname{diam}\left(O_{\min }\right)$. Given $x, y \in S_{j}$, there exists a conformal automorphism $g: S_{j} \rightarrow S_{j}$ such that $d(g(x), y) \leq \operatorname{diam}\left(O_{\min }\right)$. One may then show that there is a quasiconformal mapping $h: S_{j} \rightarrow S_{j}$ such that $h(g(x))=y, h$ is the identity off of the ball of radius $\ell\left(S_{j}\right)$ about $g(x)$, and

$$
K(h) \leq \psi_{2}^{-1}\left(\operatorname{diam}\left(O_{\min }\right)\right)+\epsilon\left(\ell\left(S_{j}\right)\right)
$$

where $\epsilon\left(\ell\left(S_{j}\right)\right) \rightarrow 0$ as $\ell\left(S_{j}\right) \rightarrow+\infty$. It follows that

$$
K\left(S_{j}\right) \leq \psi_{2}^{-1}\left(\operatorname{diam}\left(O_{\min }\right)\right)+\epsilon\left(\ell\left(S_{j}\right)\right)
$$

so that $\lim K\left(S_{j}\right)=\overline{K_{a u t}}$ as desired.

In the setting of closed surfaces it is also natural to restrict the (isotopy class of) the quasiconformal map to lie in some subgroup of the mapping class group. We recall that the mapping class group $\operatorname{Mod}(S)$ of a closed surface $S$ is the set of (isotopy classes) of selfhomeomorphisms of $S$. If $H$ is a subgroup of $\operatorname{Mod}(S)$ we say that $S$ is $H_{K}$-uniformly quasiconformally homogeneous if for any $x, y \in X$ there exists a $K$-quasiconformal homeomorphism $h: S \rightarrow S$ so that $h(x)=y$ and $[h] \in H$. (See Vlamis [28]) for a more detailed discussion.) The Torelli subgroup of $\operatorname{Mod}(S)$ is the subgroup consisting of homeomorphisms which act trivially on $H_{1}(S)$. Greenfield [19] and Vlamis [28] have independently proven:

Theorem 4.6. (Greenfield [19], Vlamis [28]) There exists $K_{\text {tor }}>1$ such that if $S$ is a closed hyperbolic surface, $H \subset \operatorname{Mod}(S)$ is the Torelli subgroup and $S$ is $H_{K}$-quasiconformally homogeneous, then

$$
K \geq K_{\text {tor }}
$$

Remark: Vlamis [28] obtains similar results for level $r$ congruence subgroups when $r \geq 3$, finite subgroups and cyclic subgroups generated by pure mapping classes.

We now turn our attention to the following question, which is motivated by Theorem 3.5.

Question 2: Does there exist a geometric characterization of uniformly quasiconformally homogeneous hyperbolic surfaces?

Proposition 3.3 guarantees that that all covers of closed hyperbolic 2-orbifolds are uniformly quasiconformally homogeneous. However, one may easily construct a quasiconformal deformation $X$ of a noncompact regular cover $S$ of a closed hyperbolic 2-orbifold $Q$ which is 
not itself a regular cover of a closed hyperbolic orbifold (see BonfertTaylor-Canary-Martin-Taylor [5, Example 5.1]). Then, since $S$ is uniformly quasiconformally homogeneous, $X$ is also uniformly quasiconformally homogeneous. (One may guarantee that $X$ is not a regular cover of a closed hyperbolic 2-orbifold by constructing it to have indiscrete length spectrum, for example.) The key difference here is that a noncompact regular cover of a closed hyperbolic 2-orbifold has an infinitedimensional quasiconformal deformation space, while a non-compact regular cover of a closed hyperbolic $n$-orbifold is quasiconformally rigid if $n \geq 3$. Therefore, the immediate generalization of Theorem 3.5 does not hold in dimension 2 .

One might then optimistically hope that every uniformly quasiconformally homogeneous surface is a quasiconformal deformation of a regular cover of a closed hyperbolic 2-orbifold. Bonfert-Taylor, Canary, Souto and Taylor [7] showed that this is not the case.

Theorem 4.7. ([7, Theorem 1.1]) There exists a uniformly quasiconformally homogeneous surface which is not a quasiconformal deformation of the regular cover of any closed hyperbolic 2-orbifold.

Sketch of proof: Given a connected countable graph $X$, each of whose vertices has valence $d \geq 3$, one may construct a hyperbolic surface $S_{X}$ by "thickening up" $X$. We first choose a compact hyperbolic surface $F$ with geodesic boundary, such that $F$ is homeomorphic to a sphere with $d$ holes and each boundary component has length 1 . One then obtains $S_{X}$ by replacing each vertex of $X$ by a copy of $F$ and gluing boundary components which correspond to the same edge of $X$.

If $\varphi$ is an automorphism of $X$, then we may construct a $L$-quasiconformal homeomorphism of $S_{X}$ which mimics $\varphi$, i.e. it takes a copy of $F$ associated to the vertex $v$ to the copy of $F$ associated to the vertex $\varphi(v)$. The quasiconformal dilatation constant $L$ depends only on our choice of $F$. Since there is a lower bound on the injectivity radius of $S_{X}$ and the diameter of each copy of $F$ is constant, we may then use a local version of Lemma 2.2 to show that if $x$ and $y$ lie in a copy of $F$, then there exists a $M$-quasiconformal homeomorphism of $S_{X}$ taking $x$ to $y$. Therefore, if the automorphism group $\operatorname{Aut}(X)$ of $X$ acts transitively on the vertices of $X$, then $S_{X}$ will be $L M$-quasiconformally homogeneous.

On the other hand, a regular cover of a closed hyperbolic 2-orbifold is quasi-isometric to the finitely generated Cayley graph of the group of deck transformations of the covering map. Therefore, since quasiconformal maps are quasi-isometries, any quasiconformal deformation of a regular cover of a closed hyperbolic 2-orbifold, is quasi-isometric to the Cayley graph of a finitely generated group. 
So, in order to construct a uniformly quasiconformally homogeneous surface which is not a quasiconformal deformation of a regular cover of a closed hyperbolic 2-orbifold, it suffices to find a connected, countable graph $X$ whose automorphism group acts transitively on its set of vertices, which is not quasi-isometric to the Cayley graph of any finitely generated group. Luckily, Eskin, Fisher and Whyte [14] showed that the Diestel-Leader graphs (see [11]) have automorphism groups that act transitively on their vertices, but are not quasi-isometric to the Cayley graph of any finitely generated group. This allows us to complete the proof.

\section{Open PROBLEMS}

Many of the open problems in the field revolve around the motivating questions from the previous section.

Question 1: Does there exists $K_{2}>1$ such that if $S$ is a uniformly quasiconformally homogeneous surface, then $K(S) \geq K_{2}$ ?

Question 2: Does there exist a geometric characterization of uniformly quasiconformally homogeneous hyperbolic surfaces? 1.

Question 2 is intriguing, but mysterious, so we will focus on Question

It follows from Theorem 3.1 and Mumford compactness, that there exists $K_{2}^{g}>1$ such that if $S$ is a closed hyperbolic surface of genus $g$, then $K(S) \geq K_{2}^{g}$.

Problem 1: Explicitly bound $K_{2}^{g}$.

If one had success with the previous problem, one might hope to find a constant which worked for all closed surfaces.

Problem 2: Can one find a bound on $K_{2}^{g}$ which is independent of $g$ ? (i.e. can one find a bound which works for all closed surfaces?)

It is natural to suspect that $K_{2}$ (assuming it exists) would be strictly less than $\bar{K}_{a u t}$.

Problem 3: Construct a uniformly quasiconformally homogeneous surface $S$ such that $K(S)<\bar{K}_{\text {aut }}$.

Returning to higher dimensions, one would like to explicitly bound $K_{n}$.

Problem 4: Explicitly bound $K_{n}$ for all $n \geq 3$. 
Manojlović-Vuorinen [25] and Vuorinen-Zhang [30] have obtained analogues of Lemma 3.6 for all $n$, with explicit bounds on the resulting functions $\psi_{n}$. One may use estimates of Adeboye-Wei [1] to obtain an explicit lower bound, for all $n$, on the diameter of any hyperbolic $n$-orbifold. Since Theorem 3.1 and McMullen's Rigidity Theorem (Theorem 3.4) imply that every quasiconformal automorphism of a uniformly quasiconformally homogeneous hyperbolic manifold is homotopic to an isometry, one should be able to follow the proof of Theorem 4.4 to produce a lower bound on $K_{n}$.

One might further hope to use the technique of proof of Theorem 4.5 to produce a sharp lower bound on $K_{n}$ in higher dimensions.

In dimension 3, there is a natural candidate for the minimal diameter hyperbolic orbifold. Gehring and Martin [15] demonstrated that, amongst all Kleinian groups containing a torsion element of order $p \geq 4$, the unique Kleinian group of minimal co-volume is a $\mathbb{Z}_{2}$-extension of the orientation preserving index 2 subgroup generated by reflections in the sides of the hyperbolic tetrahedron with Coxeter diagram $3-5-3$. Gaven Martin has conjectured:

Conjecture: (Martin) The minimum diameter hyperbolic orbifold is the $\mathbb{Z}_{2}$-extension of the orientation-preserving index 2 subgroup of the $3-5-3$ Coxeter group described above.

This leads us to:

Problem 5: Determine $K_{3}$ precisely. Is it true that if $N$ is a quasiconformally homogeneous hyperbolic 3-manifold, other than $\mathbb{H}^{3}$, then $K(N)>K_{3}$ ?

In order to adapt the proof of Theorem 4.5, one would also have to investigate an analogue of the Teichmüller extremal map in the three-dimensional setting, that is, a quasiconformal mapping of minimal distortion that maps $\Delta^{n}$ to itself, moves the origin to a point $x \neq 0$ and extends to the identity on $\partial \Delta^{n}$. However, in dimensions three and above, distortion can be measured in terms of several dilatation functions, e.g. the trace dilatation, the outer and inner dilatations, linear dilatatons, and mean dilatations (in the setting of mappings with finite distortion). The existence and uniqueness properties for such problems depend on the choice of dilatation (see Fehlmann [13]). For instance, it is known (see Kühnau [21]) that the extremal problem with boundary data for the box problem of Grötsch admits no unique solution if the dilatation is measured in terms of the inner and outer dilatation functions. Progress to date on this problem includes foundational work by Gehring and Vaisala [17] on the extremal problem in the absence of 
boundary conditions and work by Astala, Iwaneic, Martin and Onninen [3] on the extremal problem for functions of finite distortion.

\section{REFERENCES}

[1] I. Adeboye, Ilesanmi and G. Wei, "On volumes of hyperbolic orbifolds," Alg. Geom. Topol. 12(2012), 215-233.

[2] L. Ahlfors, "Quasiconformal reflections," Acta Math. 109(1963), 291-301.

[3] K. Astala, T. Iwaniec, G. Martin, J. Onninen, "Extremal mappings of finite distortion," Proc. London Math. Soc. 9(2005), 655-702.

[4] P. Bonfert-Taylor, M. Bridgeman, R.D. Canary and E. Taylor, "Quasiconformal homogeneity of hyperbolic surfaces with fixed-point full automorphisms," Math. Proc. Camb. Philos. Soc. 143(2007), 71-84.

[5] P. Bonfert-Taylor, R.D. Canary, G. Martin and E.C. Taylor, "Quasiconformal homogeneity of hyperbolic manifolds," Math. Ann. 331(2005), 281-295.

[6] P. Bonfert-Taylor, R.D. Canary, G. Martin, E.C. Taylor, and M. Wolf, "Ambient quasiconformal homogeneity of planar domains," Ann. Acad. Sci. Fenn. 35 (2010), no. 1, 275-283.

[7] P. Bonfert-Taylor, R.D. Canary, J. Souto, and E.C. Taylor, "Exotic quasiconformally homogeneous surfaces," Bull. London Math. Soc. 43(2011), 57-62.

[8] P. Bonfert-Taylor, G. Martin, A. Reid and E.C. Taylor, "Teichmüller mappings, quasiconformal homogeneity, and non-amenable covers of Riemann surfaces," P.A.M.Q. 7(2011), 455-468.

[9] P. Bonfert-Taylor and E.C. Taylor, "Quasiconformally homogeneous planar domains," Conform. Geom. Dyn. 12 (2008) 188-198.z

[10] B. Brechner and T. Erkama, "On topologically and quaisconformally homogeneous continua," Ann. Acad. Sci. Fenn. 4 (1979), 207-208.

[11] R. Diestel and I. Leader, "A conjecture concerning a limit of non-Cayley graphs," J. Alg. Comb. 14(2001), 17-25.

[12] T. Erkama, "Quasiconformally homogeneous curves", Mich. Math. J 24 (1977), 157-159.

[13] R. Fehlmann, Extremal problems for quasiconformal mappings in space, J. Anal. Math 48(1987), 179-215.

[14] D. Fisher, A. Eskin, and K. Whyte, "Coarse differentiation of quasi-isometries I: spaces not quasi-isometric to Cayley graphs," Ann. of Math. 176(2012), $221-260$.

[15] F. W. Gehring and G. J. Martin, "On the minimal volume hyperbolic 3-orbifold," Mathematical Research Letters 1 (1994), 107-114.

[16] F.W. Gehring and B. Palka, "Quasiconformally homogeneous domains," J. Anal. Math. 30(1976), 172-199.

[17] F. W. Gehring and J. Väisälä, "The coefficients of quasiconformality of domains in space," Acta Math 114(1965), 1-70.

[18] J. Gong and G. Martin, "Aspects of quasiconformal homogeneity," New Zealand J. Math. 39(2009), 117-132.

[19] M. Greenfield, "A lower bound for Torelli- $K$-quasiconformal homogeneity," preprint.

[20] G. Hjelle, "A simply connected, homogeneous domain that is not a quasidisk," Ann. Acad. Sci. Fenn. 30(2005), 135-142. 
[21] R. Kühnau, "Elementare beispiele von möglischst konformen abbildungen im dreidimensionalen raum," Wiss. Z. Martin-Luther-Univ. Halle-Wittenberg 11(1962), 729-732.

[22] F. Kwakkel and V. Markovic, "Quasiconformal homogeneity of genus zero surfaces," J. Anal. Math. 113(2011), no. 1, 173-195.

[23] P. MacManus, R. Näkki, and B. Palka, "Quasiconformally homogeneous compacta in the complex plane," Michigan Math. J. 45 (1998), 227-241.

[24] P. MacManus, R. Näkki, and B. Palka, "Quasiconformally bi-homogeneous compacta in the complex plane," Proc. L.M.S. 78(1999), 215-240.

[25] V. Manojlović and M. Vuorinen, "On quasiconformal maps with identity boundary values," Trans. A.M.S. 363(2011), 2467-2479.

[26] C.T. McMullen, Renormalization and 3-Manifolds which Fiber over the Circle, Princeton University Press, 1996.

[27] J. Sarvas, "Boundary of a homogeneous Jordan domain," Ann. Acad. Sci. Fenn. 10(1985), 511-514.

[28] N. Vlamis, "Quasiconformal homogeneity of hyperbolic surfaces and subgroups of the mapping class group," preprint.

[29] M. Vuorinen, Conformal Geometry and Quasiregular Mappings, Lecture Notes in Mathematics, 1319. Springer-Verlag, Berlin, 1988.

[30] M. Vuorinen and X. Zhang, "Distortion of quasiconformal mappings with identity boundary values," preprint.

[31] A. Yamada, "On Marden's universal constant of Fuchsian groups," Kodai Math. J. 4(1981), 266-277. 